\title{
Case Report \\ Tocilizumab Was Effective in Repairing the Large Geode in a Patient with Rheumatoid Arthritis
}

\author{
Takuya Izumiyama ${ }^{D}$, Yu Mori, and Eiji Itoi \\ Department of Orthopaedic Surgery, Tohoku University Graduate School of Medicine, 1-1 Seiryo-machi, Aoba-ku, Sendai, \\ Miyagi 980-8574, Japan \\ Correspondence should be addressed to Takuya Izumiyama; takuya-izumiyama@med.tohoku.ac.jp
}

Received 22 June 2020; Revised 9 August 2020; Accepted 10 August 2020; Published 25 August 2020

Academic Editor: Gregory J. Tsay

Copyright (c) 2020 Takuya Izumiyama et al. This is an open access article distributed under the Creative Commons Attribution License, which permits unrestricted use, distribution, and reproduction in any medium, provided the original work is properly cited.

\begin{abstract}
Rheumatoid arthritis is characterized by multiple chronic arthritis subsequently inducing joint destruction. Although subchondral geode is a well-known feature of high-disease activity, a large geode is rare. Moreover, the treatment effect of biologic agents in the repair of large geode has not been reported. The present report shows the significant effect of interleukin- 6 receptor blocker, tocilizumab, in repairing the large geode in the left humeral lateral epicondyle. This case implies that tocilizumab might be an effective treatment in patients with rheumatoid arthritis even with large geode.
\end{abstract}

\section{Introduction}

Rheumatoid arthritis (RA) is characterized by multiple chronic arthritis leading to bone erosion, joint malalignment, bony fusion, and joint destruction [1]. To prevent joint damage and decline in daily life activity, we should treat RA tightly as soon as possible because joint destruction is likely to progress in the early stage of the disease [2]. However, some patients present with bony erosion and joint destruction because of poor symptom management and/or poor prediction of severity [3]. Although subchondral geode is a well-known feature especially in high-disease activity, large geode is rare [3]. In previous studies, the large geodes mainly developed in the proximal tibia and femur [4-7], and most patients with high risk of fracture mostly underwent surgical treatment [4, 8-10]. Moreover, the treatment effect of biologic agents in the repair of large geode has been not reported. In this report, we present the significant effect of interleukin- (IL-) 6 receptor blocker, tocilizumab, in repairing the large geode in the left humeral lateral epicondyle.

\section{Case Report}

A 66-year-old Japanese male patient with bilateral elbow pain and swelling was suspected of having bone tumor in the left humeral lateral epicondyle by radiography in an orthopedic clinic and referred to our department. However, bone tumor was denied by experienced bone and soft tissue tumor surgeons with magnetic resonance imaging (MRI). The subjective symptoms were swelling and pain of the bilateral elbow and left wrist. The left elbow had deformity with neither redness nor local heat. The laboratory examination showed erythrocyte sedimentation rate of $94 \mathrm{~mm}$, C-reactive protein level of $7.0 \mathrm{mg} / \mathrm{dL}$, rheumatoid factor of $70.2 \mathrm{IU} / \mathrm{dL}$, and anticyclic citrullinated peptide antibody level of $156.8 \mathrm{U} / \mathrm{mL}$. Plain radiography showed a $29.7 \mathrm{~mm} \times 21.0 \mathrm{~mm}$ of large geode area in the left humeral lateral epicondyle (Figure 1). MRI demonstrated a large lesion, which had high intensity in T2-weighted images, low intensity in T1-weighted images, and enhanced peripheral area of the geode in gadoliniumenhanced T1-weighted images (Figure 2). These images suggested that the cyst consisted of fluid with peripheral synovitis. The patient fulfilled 2010 ACR/EULAR 


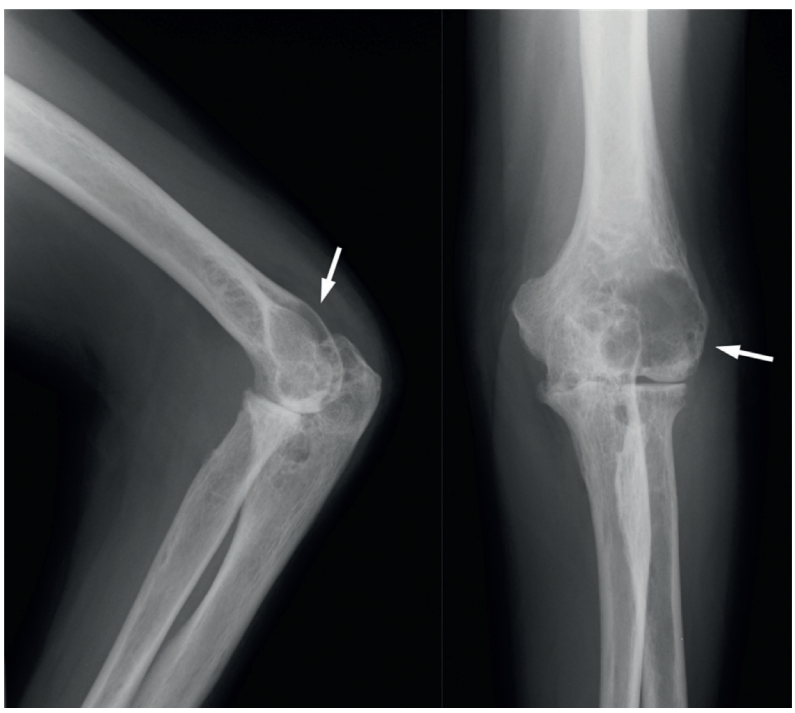

Figure 1: Plain radiograph of the left elbow obtained at the first visit to our department. A large transparent area is observed in the posterior lateral humeral bone (arrow). The vertical range is $29.8 \mathrm{~mm}$, and lateral range is $33.0 \mathrm{~mm}$ in the anteroposterior view.

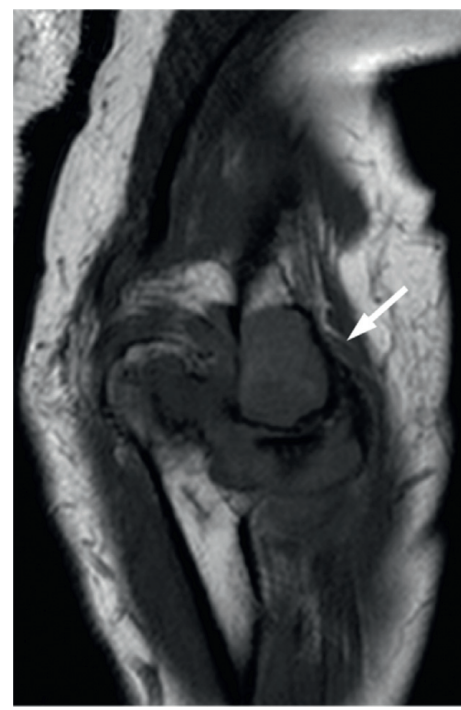

T1WI

(a)

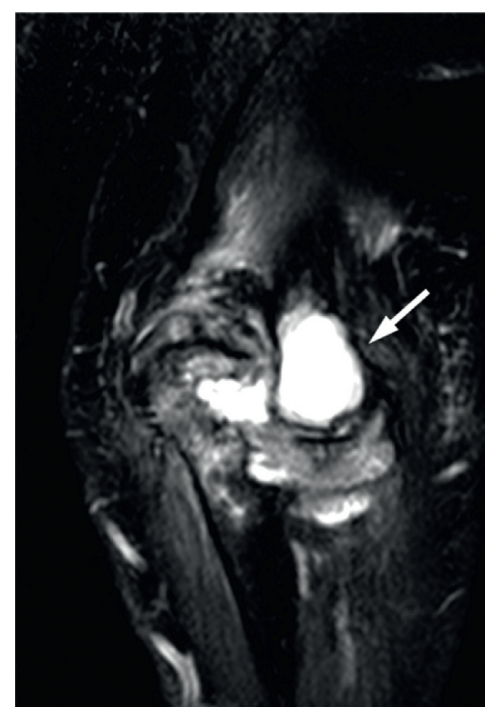

T2WI-Fs

(b)

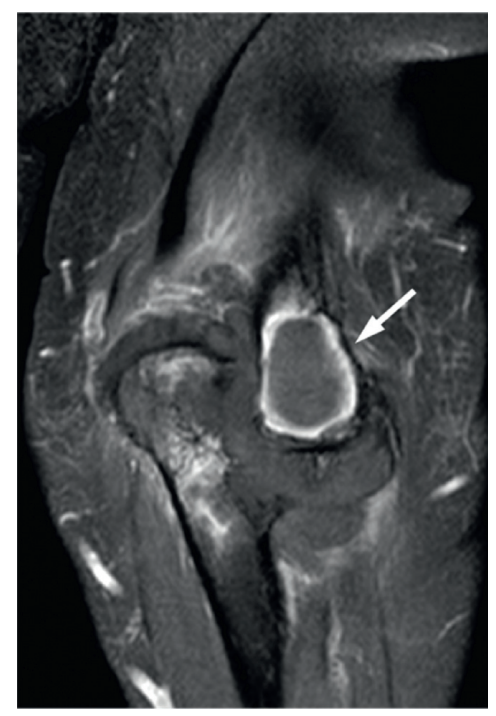

Gd-T1WI-Fs

(c)

Figure 2: Magnetic resonance imaging of the left elbow obtained at the first visit to our department. (a) T1-weighted image (T1WI); (b) fatsuppressed T2-weighted image (T2WI-Fs); and (c) gadolinium-enhanced and fat-suppressed T1-weighted image (Gd-T1WI-Fs). A large low-intensity area is observed in T1WI, and high-intensity area is observed in T2WI-Fs. In Gd-T1WI-Fs, a large low-intensity area enhanced peripherally is noted (arrow). It seems as a large cyst surrounded by synovium.

classification criteria for RA [11] and was diagnosed with RA. Methotrexate was administered, and the dose was gradually increased to $12 \mathrm{mg} /$ week. However, the disease activity score (DAS) 28 was still high (Figure 3), and radiography indicated no evident improvement of the large geode in the left humeral lateral epicondyle (Figure 4). Therefore, $162 \mathrm{mg}$ of tocilizumab was administered every 2 weeks. After subcutaneous injection of tocilizumab, the patient reported articular pain, laboratory data showed inflammation factor, and DAS28 immediately improved (Figure 3). Furthermore, the large geode in the left humeral lateral epicondyle gradually improved, and progression of bone formation from peripheral area was shown in radiography (Figure 5). The area of geode was significantly decreased to $10.8 \mathrm{~mm} \times 8.3 \mathrm{~mm}$. As additional treatment, denosumab was administered by subcutaneous injection every three months. However, additional denosumab showed no eminent effect on geode repair (Figure 6). In contrast, MRI showed decreased area in the geode and diminished peripheral contrast enhancement effect compared to MRI before tocilizumab administration (Figure 7). Left elbow pain significantly ameliorated, and activity of daily life improved without surgical treatment. 


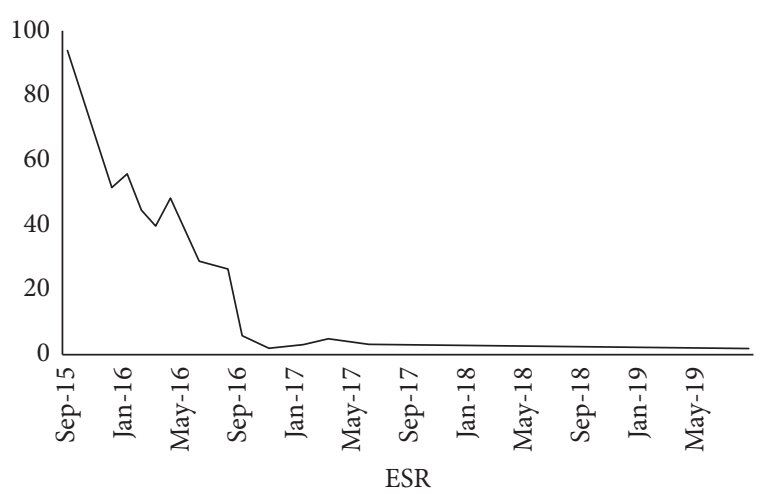

(a)

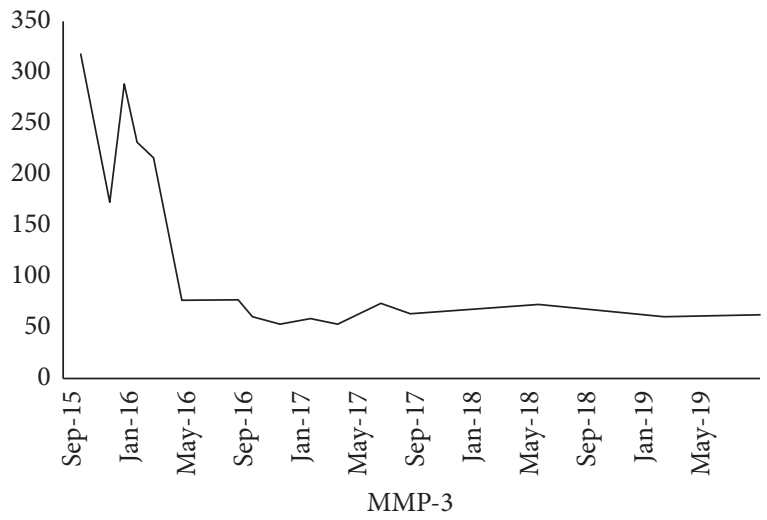

(c)

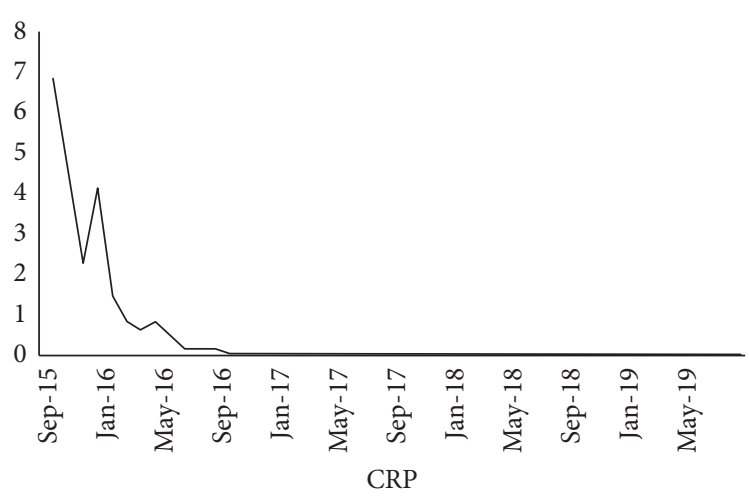

(b)

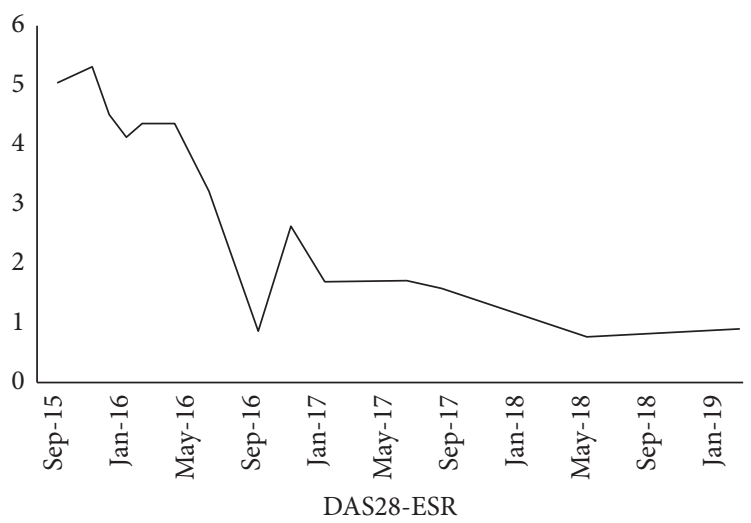

(d)

FIgURE 3: Transition of (a) erythrocyte sedimentation rate (ESR), (b) C-reactive protein (CRP), (c) matrix metalloproteinase (MMP)-3, and (d) disease activity score (DAS) 28. After visiting our department, the patient was treated with methotrexate. From August 22, 2016, the patient started to receive tocilizumab, and all laboratory data and DAS28 improved remarkably and immediately after administration.

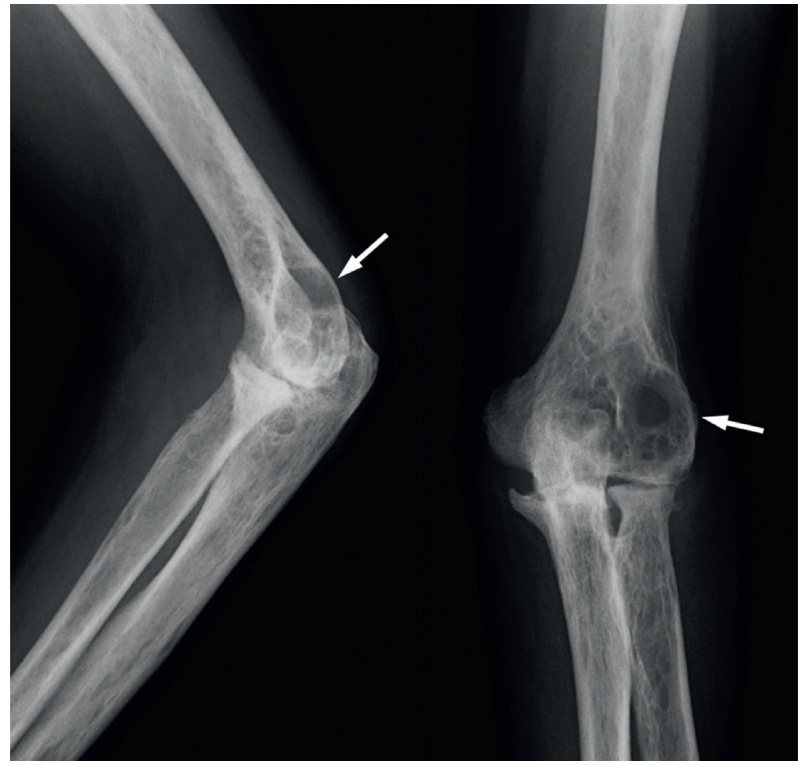

Figure 4: Plain radiograph of the left elbow at 10 months since the start of oral methotrexate. The large transparent area still remained, and poor repair is noted in the posterior lateral humeral bone (arrow).

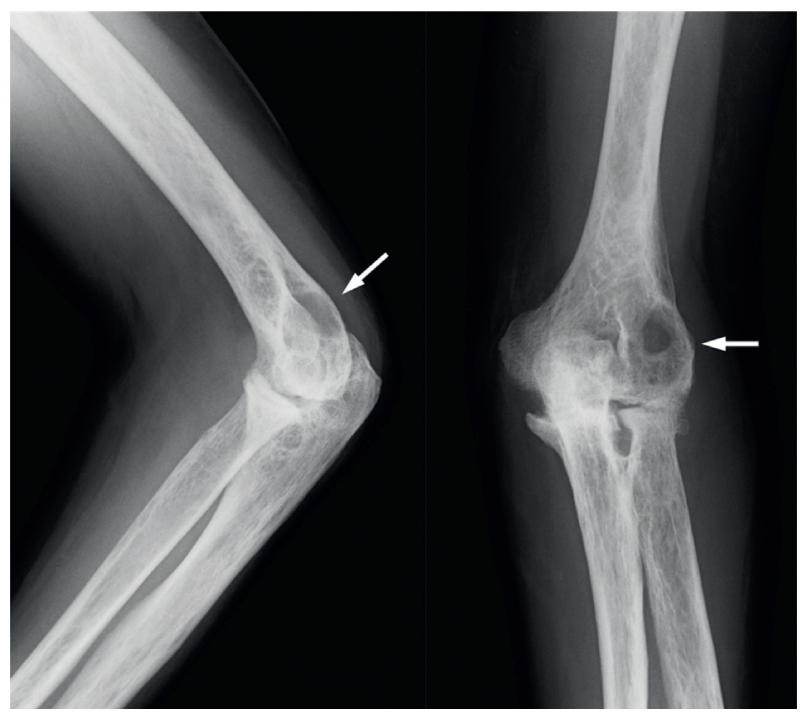

FIGURE 5: Plain radiograph of the left elbow at 10 months since the administration of subcutaneous tocilizumab injection. The transparent area gradually decreased, and bone formation progressed from the peripheral area (arrow). The size of evident geode is $10.8 \mathrm{~mm} \times 8.3 \mathrm{~mm}$. 


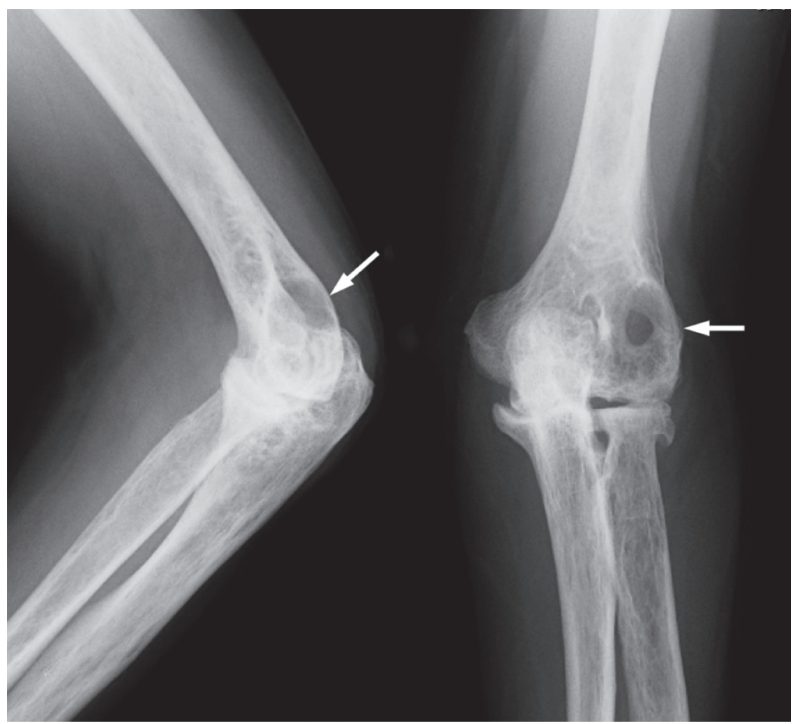

Figure 6: Plain radiograph of the left elbow at one month after administration of denosumab every three months. The transparent area showed little improvement effect compared to Figure 5 (arrow).

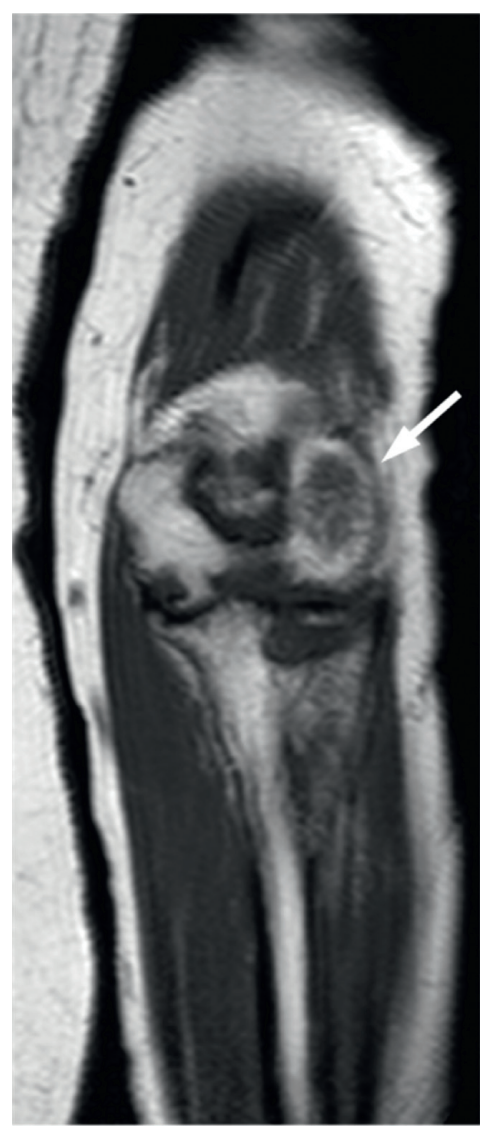

T1WI

(a)

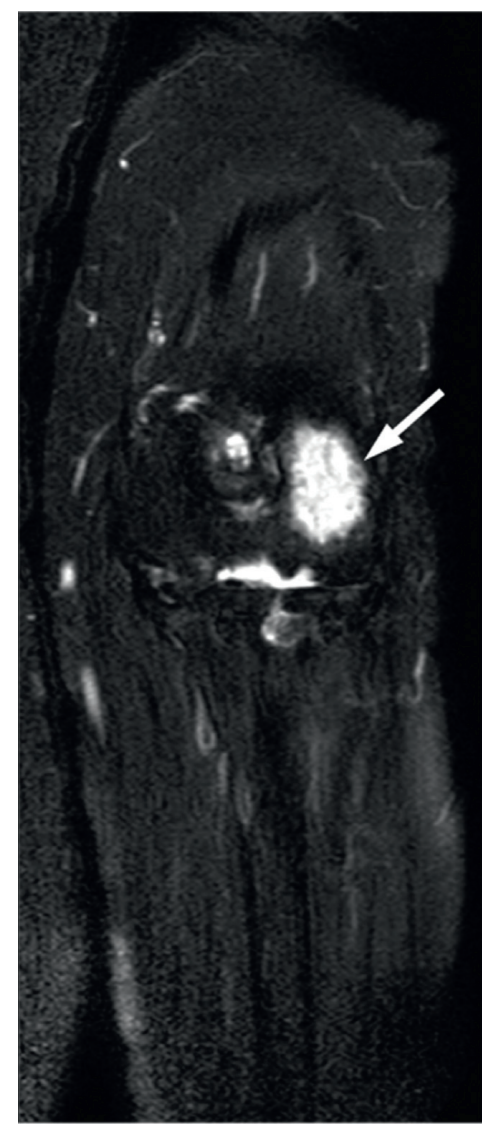

T2WI-Fs

(b)

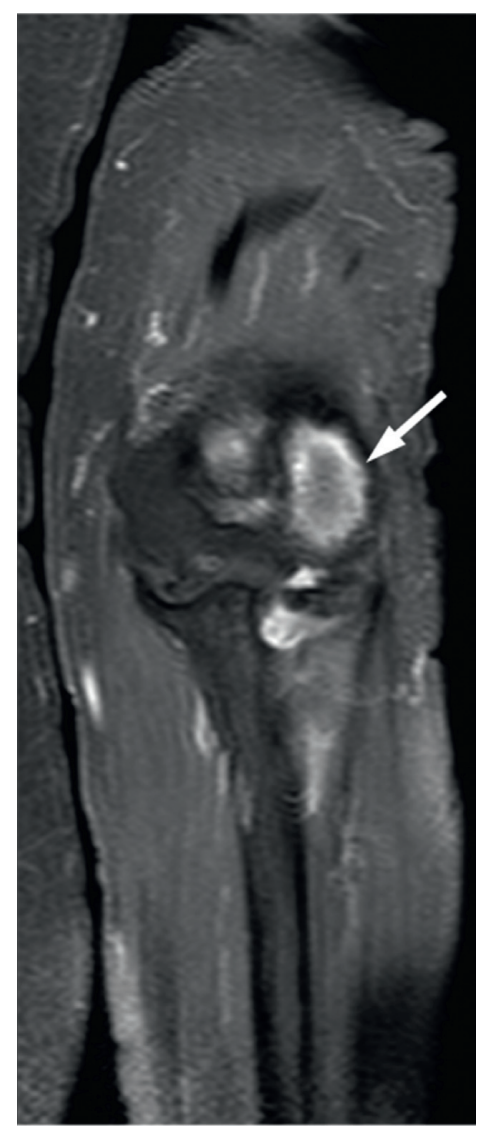

Gd-SPIR

(c)

Figure 7: Magnetic resonance image of the left elbow, 3 years 5 months after the first visit to our department. (a) T1-weighted image (T1WI); (b) fat-suppressed T2-weighted image (T2WI-Fs); and (c) gadolinium-enhanced short T1 inversion recovery image (Gd-SPIR). The highintensity area appeared from the peripheral cavity and internal low-intensity area became slightly brighter in T1WI. The peripheral highintensity area was considered to demonstrate replaced bone marrow fat. The high-intensity area is noted in T2WI-Fs, but the size and signal strength decreased. In Gd-SPIR, the low-intensity area became slightly brighter, and the peripherally enhanced area seemed to be weaker (arrow). 


\section{Discussion}

This case showed eminent repair of the large geode by tocilizumab in a patient with RA. Several reports revealed that biological disease-modifying antirheumatic drugs (DMARDs) could repair the bone erosion [12-15]. However, to the best of our knowledge, the treatment effect on the large geode has not been reported. Although several previous studies described the large geode in RA $[9,10,16]$, surgeries were performed immediately after diagnosis because of existing fracture or preventing the risk of fracture $[9,10,16,17]$. Tocilizumab administration may be an effective treatment for large geode and one of the useful treatment alternatives before performing surgeries.

Our strong interest is whether tocilizumab has an additional treatment effect other than anti-inflammatory effect. The positive effect for bone metabolism related with anti-IL-6 therapy is still unclear; nevertheless, some studies showed that tocilizumab increases bone formation marker and decreases bone resorption marker [18-22]. Kume et al. showed the improvement effect on bone mineral density (BMD) in patients with RA treated with tocilizumab [23]. Furthermore, Chen et al. showed the decrease of the bone resorption marker and BMD improvement with tocilizumab treatment [24]. These antibone resorption effects of tocilizumab may explain the repairing effect of the large geode. Denosumab indicated the effect of the bone erosion repair by strongly inhibiting osteoclast activity [25]; therefore, we added denosumab for the acceleration of bone repair. However, expected effects were not obtained in this case. These results suggested that suppressing inflammatory synovitis might be more effective in geode repair compared to inhibiting osteoclast activity in patients with RA with large geode.

Another question is whether other biological DMARDs, including anti-tumor necrosis factor- (TNF-) $\alpha$ and antiCD80/86, have the same improvement effect on the large geode. Several studies revealed that anti-TNF- $\alpha$ improves bone metabolism and BMD $[26,27]$. A few reports showed the positive effect of anti-CD80/86 treatment on bone metabolism $[28,29]$. Biological DMARDs potentially have the positive effect on bone effect repair. However, to our best knowledge, similar studies have not been published.

Therefore, we showed the eminent effect of tocilizumab on the large geode repair. Considering tocilizumab as an alternative treatment for patients with large geode may be helpful in the prevention of fracture, and consequently, patients with RA may have the chance of maintaining activities of daily living.

\section{Data Availability}

All data generated or analyzed during this study are included in this published article.

\section{Conflicts of Interest}

The authors declare that they have no conflicts of interest.

\section{References}

[1] J. S. Smolen, D. Aletaha, M. Koeller, M. H. Weisman, and P. Emery, "New therapies for treatment of rheumatoid arthritis," The Lancet, vol. 370, no. 9602, pp. 1861-1874, 2007.

[2] J. S. Smolen, D. Aletaha, J. W. J. Bijlsma et al., "Treating rheumatoid arthritis to target: recommendations of an international task force," Annals of the Rheumatic Diseases, vol. 69, no. 4, pp. 631-637, 2010.

[3] D. Rabar, P. Crozes, M. Lernoud, and F. Meignan, "Large geodes in rheumatoid arthritis without joint destruction," Joint Bone Spine, vol. 69, no. 6, pp. 617-621, 2002.

[4] D. Schapira, "Large subarticular cysts (geodes): an unusual finding," The Israel Medical Association Journal: IMAJ, vol. 2, no. 7 , p. $562,2000$.

[5] M. I. Jayson, A. S. Dixon, and P. Yeoman, "Unusual geodes ("bone cysts") in rheumatoid arthritis," Annals of the Rheumatic Diseases, vol. 31, no. 3, pp. 174-178, 1972.

[6] D. Resnick, G. Niwayama, and R. Coutts, "Subchondral cysts (geodes) in arthritic disorders: pathologic and radiographic appearance of the hip joint," American Journal of Roentgenology, vol. 128, no. 5, pp. 799-806, 1977.

[7] B. F. Morrey, "Rheumatoid pseudocyst (geode) of the femoral neck without apparent joint involvement," Mayo Clinic Proceedings, vol. 62, no. 5, pp. 407-411, 1987.

[8] H. Yamanaka, K. Goto, and M. Suzuki, "Total knee arthroplasty for rheumatoid arthritis patients with large tibial condyle defects," Journal of Orthopaedic Surgery, vol. 20, no. 2, pp. 148-152, 2012.

[9] A. Jaiswal, R. Thakur, J. Relwani, and W. Ogufere, "Spontaneous displacement of olecranon fracture through geode salvaged by elbow replacement," Rheumatology International, vol. 30, no. 6, pp. 785-787, 2010.

[10] M. M. Maher, J. Kennedy, D. Hynes, J. G. Murray, and D. O'Connell, "Giant distal humeral geode," Skeletal Radiology, vol. 29, no. 3, pp. 165-167, 2000.

[11] D. Aletaha, T. Neogi, A. J. Silman et al., "2010 Rheumatoid arthritis classification criteria: an American College of Rheumatology/European League against Rheumatism collaborative initiative," Arthritis \& Rheumatism, vol. 62, no. 9, pp. 2569-2581, 2010.

[12] S. Finzel, J. Rech, S. Schmidt et al., "Repair of bone erosions in rheumatoid arthritis treated with tumour necrosis factor inhibitors is based on bone apposition at the base of the erosion," Annals of the Rheumatic Diseases, vol. 70, no. 9, pp. 1587-1593, 2011.

[13] S. Finzel, S. Kraus, C. P. Figueiredo et al., "Comparison of the effects of tocilizumab monotherapy and adalimumab in combination with methotrexate on bone erosion repair in rheumatoid arthritis," Annals of the Rheumatic Diseases, vol. 78, no. 9, pp. 1186-1191, 2019.

[14] S. Ros-Expósito, J. M. Ruiz-Martín, P. Sanz-Frutos, and D. De La Fuente De Dios, "Bone erosion repair with adalimumab in rheumatoid arthritis," Clinical Rheumatology, vol. 29, no. 11, pp. 1339-1340, 2010.

[15] C. Lukas, D. van der Heijde, S. Fatenajad, and R. Landewe, "Repair of erosions occurs almost exclusively in damaged joints without swelling," Annals of the Rheumatic Diseases, vol. 69, no. 5, pp. 851-855, 2010.

[16] B. P. Wordsworth, A. G. Mowat, and N. A. Watson, "Fracture through a geode in the proximal ulna," Rheumatology, vol. 23, no. 2, pp. 110-112, 1984. 
[17] N. Nakagawa, S. Abe, Y. Saegusa et al., "Giant geode at the olecranon in the rheumatoid elbow? Two case reports," Clinical Rheumatology, vol. 23, no. 4, pp. 358-361, 2004.

[18] E. Terpos, K. Fragiadaki, M. Konsta, C. Bratengeier, A. Papatheodorou, and P. P. Sfikakis, "Early effects of IL-6 receptor inhibition on bone homeostasis: a pilot study in women with rheumatoid arthritis," Clinical and Experimental Rheumatology, vol. 29, no. 6, pp. 921-925, 2011.

[19] P. Garnero, E. Thompson, T. Woodworth, and J. S. Smolen, "Rapid and sustained improvement in bone and cartilage turnover markers with the antiâinterleukin-6 receptor inhibitor tocilizumab plus methotrexate in rheumatoid arthritis patients with an inadequate response to methotrexate: results from a substudy of the multicenter double-blind, placebocontrolled trial of tocilizumab in inadequate responders to methotrexate alone," Arthritis \& Rheumatism, vol. 62, no. 1, pp. 33-43, 2010.

[20] M. A. Karsdal, G. Schett, P. Emery et al., "IL-6 receptor inhibition positively modulates bone balance in rheumatoid arthritis patients with an inadequate response to anti-tumor necrosis factor therapy: biochemical marker analysis of bone metabolism in the tocilizumab RADIATE study (NCT00106522)," Seminars in Arthritis and Rheumatism, vol. 42, no. 2, pp. 131-139, 2012.

[21] N. Nishimoto, K. Yoshizaki, N. Miyasaka et al., "Treatment of rheumatoid arthritis with humanized anti-interleukin- 6 receptor antibody: a multicenter, double-blind, placebo-controlled trial," Arthritis \& Rheumatism, vol. 50, no. 6, pp. 1761-1769, 2004.

[22] A. C. Bay-Jensen, A. Platt, I. Byrjalsen, P. Vergnoud, C. Christiansen, and M. A. Karsdal, "Effect of tocilizumab combined with methotrexate on circulating biomarkers of synovium, cartilage, and bone in the LITHE study," Seminars in Arthritis and Rheumatism, vol. 43, no. 4, pp. 470-478, 2014.

[23] K. Kume, K. Amano, S. Yamada et al., "The effect of tocilizumab on bone mineral density in patients with methotrexate-resistant active rheumatoid arthritis," Rheumatology, vol. 53, no. 5, pp. 900-903, 2014.

[24] Y.-M. Chen, H.-H. Chen, W.-N. Huang et al., "Tocilizumab potentially prevents bone loss in patients with anticitrullinated protein antibody-positive rheumatoid arthritis," PLoS One, vol. 12, no. 11, Article ID e0188454, 2017.

[25] S. B. Cohen, R. K. Dore, N. E. Lane et al., "Denosumab treatment effects on structural damage, bone mineral density, and bone turnover in rheumatoid arthritis: a twelve-month, multicenter, randomized, double-blind, placebo-controlled, phase II clinical trial," Arthritis \& Rheumatism, vol. 58, no. 5, pp. 1299-1309, 2008.

[26] G. Dischereit, I. H. Tarner, U. Müller-Ladner, and U. Lange, "Infliximab improves bone metabolism and bone mineral density in rheumatoid arthritis and ankylosing spondylitis: a prospective 2-year study," Clinical Rheumatology, vol. 32, no. 3, pp. 377-381, 2013.

[27] C. Gabay, P. Emery, R. van Vollenhoven et al., "Tocilizumab monotherapy versus adalimumab monotherapy for treatment of rheumatoid arthritis (ADACTA): a randomised, doubleblind, controlled phase 4 trial," The Lancet, vol. 381, no. 9877 , pp. 1541-1550, 2013.

[28] S. Roser-Page, T. Vikulina, M. Zayzafoon, and M. N. Weitzmann, "CTLA-4Ig-induced T cell anergy promotes wnt-10b production and bone formation in a mouse model," Arthritis \& Rheumatology, vol. 66, no. 4, pp. 990-999, 2014.
[29] M. Tada, K. Inui, Y. Sugioka et al., "Delayed wound healing and postoperative surgical site infections in patients with rheumatoid arthritis treated with or without biological disease-modifying antirheumatic drugs," Clinical Rheumatology, vol. 35, no. 6, pp. 1475-1481, 2016. 